\title{
Do extended effective-medium formulas scale properly?
}

\author{
Craig F. Bohren \\ Department of Meteorology, Pennsylvania State University, University Park, PA 16802 \\ bohren@meteo.psu.edu
}

\begin{abstract}
The original Bruggeman and Maxwell Garnett formulas for the average dielectric function of a composite medium satisfy a scaling law: if the dielectric functions of both components are scaled by the same factor, the average dielectric function scales by this factor. These formulas are independent of the size of polarizable elements. Extended formulas explicitly account for size, but at least one formula fails to satisfy the scaling law, a likely failing of all such formulas.
\end{abstract}

Keywords: Bruggeman formula, composite materials, effective medium, Maxwell Garnett formula.

Theories of effective or average dielectric functions of composite media, which could include mixtures of gases, liquids, and powders, as well as suspensions of small particles, go back at least 200 years. The literature on this subject is voluminous and dispersed among many fields, and hence I do not review it here. For classical and modern papers, see the compendium edited by Lakhtakia [1].

For my purpose here the fundamental problem is to determine how the average dielectric function $\epsilon_{a v}$ of a two-component composite particulate medium depends on the dielectric functions of its components, denoted by $\epsilon_{m}$ and $\epsilon$, and their relative amounts (often specified by volume fractions). Each component is composed of regions, possibly disconnected, that are coherent aggregations of sufficiently many molecules that they can be assigned macroscopic properties. Disconnected regions (particles) must be electrically small, that is, small compared with all relevant wavelengths. The medium is inhomogeneous with a spatially varying dielectric function $\epsilon(\mathbf{x})$ having only two values. Because of the linearity of the field equations and the definition of $\epsilon_{a v}=\langle\epsilon(\mathbf{x}) \mathbf{E}(\mathbf{x})\rangle /\langle\mathbf{E}(\mathbf{x})\rangle$ in terms of spatial averages over sufficiently large volumes, any effective-medium theory (homogenization formula) in the long-wavelength limit must satisfy the requirement that for arbitrary $p$ if $\epsilon_{m} \rightarrow p \epsilon_{m}$ and $\epsilon \rightarrow p \epsilon$, then $\epsilon_{a v} \rightarrow p \epsilon_{a v}$. This scaling law seems not to be widely recognized, although it was known to Wiener [2], who called it "the theorem about the unaltered ratio of constants" (see, Michel [3]).

The two most widely used average dielectric function formulas are the Maxwell Garnett formula

$$
\frac{\epsilon_{a v}-\epsilon_{m}}{\epsilon_{a v}+2 \epsilon_{m}}=f \frac{\epsilon-\epsilon_{m}}{\epsilon+2 \epsilon_{m}}
$$

where $f$ is the volume fraction of the component with dielectric function $\epsilon$, and the symmetric Bruggeman formula

$$
f \frac{\epsilon-\epsilon_{a v}}{\epsilon+2 \epsilon_{a v}}+(1-f) \frac{\epsilon_{m}-\epsilon_{a v}}{\epsilon_{m}+2 \epsilon_{a v}}=0
$$


both of which scale and are independent of the size of the components.

In recent years, however, extended effective-medium formulas, ones in which the size of polarizable elements appears explicitly, have been proposed. Ruppin [4] examines some of these formulas in light of various criteria. Perhaps the easiest formula to understand is an extended Maxwell Garnett formula, which stems from the Clausius-Mossotti relation. The Maxwell Garnett formula in its original form applies to spheres with dielectric function $\epsilon$ suspended in a continuous medium with dielectric function $\epsilon_{m}$, and the polarizability of the spheres is the expression from electrostatics. But for spheres we can use instead an unrestricted expression for the electric dipole term from the Lorenz-Mie series for scattering by an arbitrary homogeneous sphere of radius $a$ illuminated by a plane wave. The result is

$$
\epsilon_{a v}=\epsilon_{m}\left(\frac{1+3 i f \frac{a_{1}}{x^{3}}}{1-\frac{3}{2} i f \frac{a_{1}}{x^{3}}}\right) .
$$

The size parameter $x=2 \pi a \sqrt{\epsilon_{m}} / \lambda$, where $\lambda$ is the free-space wavelength, can be complex. Because the scattering coefficient $a_{1}$ satisfies $\left|a_{1}\right| \leq 1$ for all $\epsilon, \epsilon_{m}$, and $a$, $a_{1} / x^{3}$ is always finite, but Eq. (3) can satisfy the scaling law only if $a_{1} \propto x^{3}$; this scattering coefficient depends on $x$ and $m x$, where $m=\sqrt{\epsilon / \epsilon_{m}}$ (the permeabilities of the sphere and continuous medium are assumed to be equal). Although $m$ is invariant, $x$ is not. Moreover, $a_{1}$ is a function of sines and cosines of $x$ and $m x$. Thus whatever merits Eq. (3) may possess, it does not scale except in the limit $x \rightarrow 0$.

I chose this extended effective-medium formula because of the ease with which it can be shown to not satisfy the scaling law. Analytical proofs for more complicated formulas may not be possible, and hence one would have to resort to computations to show that they do or do not scale. I conjecture, however, that all extended effective-medium theories do not. The electrical smallness requirement is indefinite, whereas an extended effective-medium formula either satisfies the scaling law or it does not, and one can determine quantitatively the degree to which it does not.

The only justification for extending effective-medium theories to include a size dependence is to describe reality more faithfully, to obtain better agreement with the widest class of measurements of likely interest, not just, say, transmission measurements. Given a few adjustable parameters (such as size distribution) one can fit any single kind of measurement. Benefits in extending effective-medium theories may be accompanied by costs, but it is not possible to say a priori if and under what circumstances the benefits exceed the costs and by how much. Nevertheless the failure of extended effective-medium theories to satisfy a scaling law at the very least signals caution in their use. Moreover, the scaling law can be used to compare different extended effective-medium theories. All else being equal, the closer an extended effective-medium theory comes to satisfying the scaling law the better it is.

\section{References}

[1] A. Lakhtakia, Ed., Selected Papers on Linear Optical Composite Materials, SPIE Optical Engineering Press, Bellingham, WA(1996). 
[2] O. Wiener, "Die Theorie des Mischkörpers für das Feld der Stationären Strömung. Erste Abhandlung: Die Mittelwertsätze für Kraft, Polarisation und Energie," Abh. Math.-Phys. Kl. Königl. Sächs. Ges. Wissen. 32, 507-604 (1912).

[3] B. Michel, "Theory of the mixed body in an electrostatic field," in Selected Papers on Linear Optical Composite Materials, A. Lakhtakia, Ed., pp. 352-357, SPIE Optical Engineering Press, Bellingham, WA(1996).

[4] R. Ruppin, "Evaluation of extended Maxwell-Garnett theories," Opt. Commun. 182, 273-279 (2000) [doi:10.1016/S0030-4018(00)00825-7].

Craig F. Bohren is Distinguished Professor Emeritus of Meteorology, Pennsylvania State University. He is the co-author (with Donald R. Huffman) of Absorption and Scattering of Light by Small Particles and (with Eugene E. Clothiaux) of Fundamentals of Atmospheric Radiation. 